\title{
Edible oil electrical parameters detection system design based on interdigital sensor
}

\author{
Yufeng Peng ${ }^{1, a}$, Lili Zhao ${ }^{1, b}$ and Shaoge Yang ${ }^{1, c}$ \\ ${ }^{1}$ College of Physics and Information Engineering, Henan Normal University,46 Jianshe \\ Avenue,P.R.China 453000 \\ ayufengp@htu.cn,,1061869700@qq.com, cyang shaoge2008@163.com
}

Keywords: interdigital capacitance sensor ;edible oil detection ;AD7746 ; PIC18F452

\begin{abstract}
Edible oil quality is related to people healthy,existing technology can't realize the convenient and fast detection.This paper studied on the basis of the interdigital capacitance sensor,based on the dielectric constant measurement principle ,used to detect edible oil electrical parameter.Useing capacitance AD7746 digital conversion chip,at the same time AD7746 utilizes $\mathrm{I}^{2} \mathrm{C}$ serial interface protocol and communicates with micro controller PIC18F452 single chip, achieving capacitance data acquisition and processing, form a shortcut edible oil detection system of high accuracy in real time.
\end{abstract}

\section{Introduction}

Edible oil quality in relation to thousands of households, illegal cooking oil and repeatedly fried oil seriously affect people's health.Long-term consumption could lead to a series of diseases. Illegal cooking oil rancidity indicators far beyond the provisions of the state,at the same time illegal cooking oil contains a lot of aflatoxin,all of these greatly increase the polarized molecules in the oil.And the oil which repeatedly fried in high temperature environment will produce a series of chemical reactions and generate lipid oxide,dimers, hydrogenated oxide.The component of oil dieletric polarization directly affects the size of the dielectric constant,the more of the dielectric polarization the greater of the dielectric constant.

Traditional methods include sensory evaluation method,column chromatography,chemicalsystem, spectrum,infrared detection method.These methods are time-consuming and the extensive use of chemical reagents also cause some pollution on the environment.At present,one of the rapid detection is the measurement of polarization component content.But the difficulty in detecting a capacitance of the dielectric constant is that the high-performanceCfron, low cost and small parasitic capacitance, anti-interference abilityt of the front capacitance sensor signal processing performance.Therefore, this paper attempts to design a circular interdigital capacitance sensor and cooperate with the corresponding hardware circuit, to accomplish the realization of edible oil quality testing.

\section{New type capacitance sensor}

The capacitance sensor has advantages in that it is simple in construction and is highly sensitive. The capacitance sensor is not a probe-type but rather a plate-type so that the large area can reduce the effect of roughness in the measured object.Capacitive sensor principle is relatively simple,capacitive sensor is a capacitor with variable parameters, in most cases,capacitive sensors which is based on the air as a medium is a parallel plate capacitor consisted of two parallel metal plates. Set for the sensor capacitance $\mathrm{C}, \mathrm{F} ; \varepsilon_{\mathrm{r}}$ is the dielectric constant of medium between the two 
plates, $\mathrm{F} / \mathrm{m} ; \mathrm{S}$ is the plate area, $\mathrm{m}^{2} ; \varepsilon_{\mathrm{r}}$ as the relative dielectric constant; $\varepsilon_{0}$ as the vacuum dielectric constant $\left(8.8542 \times 10^{-12} \mathrm{~F} / \mathrm{m}\right)$. Without considering the edge effect parallel plate capacitor capacitance is calculated as:

$$
C=\frac{\varepsilon S}{d}=\frac{\mathcal{E}_{\mathrm{r}} \boldsymbol{\varepsilon}_{0} S}{d}
$$

Parallel plate capacitance sensor has poor stability, large amount of drift,large amount of the error of detection capacitance,and is difficult to measure a tiny change in edible oil capacitance.Choose bipolar plate area to reach a certain level, this has led to inconvenient measure.To satisfy high sensitivity, a certain stability, sensor size to facilitate measurement requirements, reduce edge effects, a circular interdigital capacitance sensor is designed.As shown in figure 1. Figure 1 shows the sensor build in double-sided copper clad laminate as well as the shape of the designed capacitance sensor plate. The base material of the sensor is epoxy resin,and it is coated with a copper layer $0.8 \mathrm{~mm}$ thick. The copper layer is then cut to the desired pattern through an etching process. The radius of the circular interdigital capacitance sensor is $20 \mathrm{~mm}$ which are maximized sizes to achieve more sensitivity.

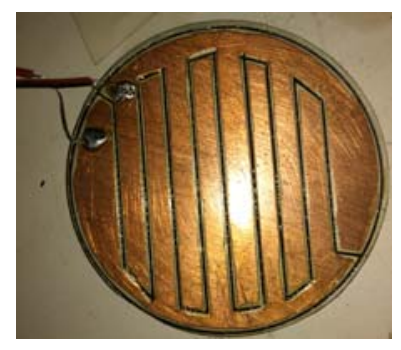

Fig.1 Structure diagram of interdigital sensor

When several of the same capacitor plate formed alternately, by the distribution principle of electric field line could be known

$$
\begin{aligned}
& q=\iint_{s} \sigma d s=\int_{\frac{d}{2}}^{\frac{b}{2}} \frac{\varepsilon_{0} U}{\pi x} d x=\frac{l \varepsilon_{0} U}{\pi} \int_{\frac{d}{2}}^{\frac{b}{2}} \frac{d x}{x}=\frac{l \varepsilon_{0} U}{\pi} \ln \left(\frac{b}{d}\right) \\
& C=\frac{q}{U}=\frac{l \varepsilon_{0} U}{\pi} \ln \left(\frac{b}{d}\right)
\end{aligned}
$$

1 is the sum of the length of the interdigital, the plate width is b, the bipolar plate spacing is d.As shown in figure 2.

In the actual capacitance of capacitive sensor can be expressed as

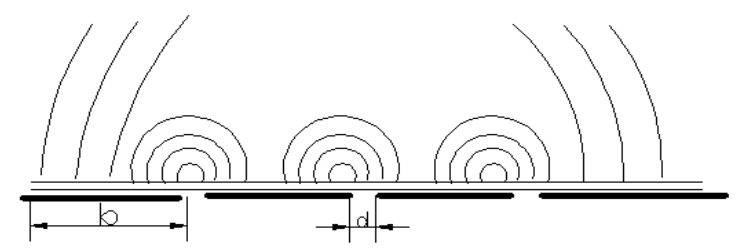

Fig.2 The sketch of electric line of interdigital sensor

$$
C=C_{h}+C_{i}
$$




$$
\begin{aligned}
& C_{h}=q / U=\frac{l \varepsilon_{0} U}{\pi} \ln \left(\frac{b}{d}\right) \\
& C_{i}=q / U=\frac{l \varepsilon U}{\pi} \ln \left(\frac{b}{d}\right)
\end{aligned}
$$

$\mathrm{C}$ refers to the total capacitance; $\mathrm{C}_{\mathrm{h}}$ refers to the capacitance formed by air medium, $\mathrm{C}_{\mathrm{i}}$ refers to the capacitance which regards the dielectric constant $\varepsilon$ printing plate upholder as the medium[1].

In the actual measurements, if you put the circular interdigital sensor into the oil groove, the $\varepsilon_{0}$ will change and the capacitance $\mathrm{C}$ in the sensorwill change alike.In the application, choosing 0.8 $\mathrm{mm}$ thick double-sided copper clad laminate, copper clad laminate middle is a small temperature coefficient of non-metallic materials,copper clad laminate with laser cutting technology, engraving the plate spacing. Because it is a double-sided copper clad laminate, the other side is copper, playing a role in protecting the plate, against external electromagnetic interference.

\section{The design of the detection circuit}

In general, in order to improve the measurement accuracy and sensitivity, we will add a certain amount of artificial circuit and hardware circuit, and then the system stray capacitance will increases with a large number of discrete component used.This paper use of integrated circuit chip capacitor detection chip AD7746, together with the corresponding peripheral circuits. And using PIC18F452 microcontroller for control and data processing.

AD7746 Introduction. The AD7746 has 24-bit $\Sigma-\Delta$ technology, voltage reference and the clock generator, and the built-in programmable compensation circuit.Structure is shown in figure .The AD7746 has two channels. Each channel can be configured as single-ended or differential. The AD7746 are designedfor floating capacitive sensors. The ad7746 achieves the conversion between the variable capacitor and the fixed input voltage, where the input voltage is a fixed chip voltage excitation $(250 \mathrm{kHz}$ square wave signal), the variable capacitor is the capacitance under test [2].As shown in figure 3.

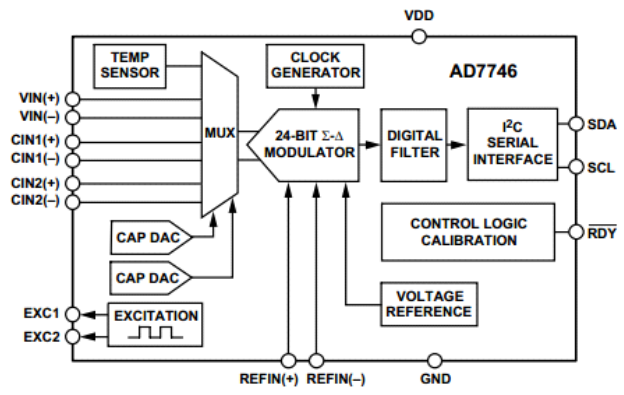

Fig.3 Principle diagram of AD7746

The capacitor which is under test can be connected to AD7746 directly, the measured capacitance value which is converted into a digital quantity can be stored in three consecutive registers.Measuring range of the variable capacitance is $\pm 4 \mathrm{pF}$, can be set by the on-chip programmable compensation offset 0 a $17 \mathrm{pF}[3]$.

AD7746 uses the I2C serial interface, can easily be controlled and corrected by single chip microcomputer.AD7746 has total of 19 registers,among them,the capacitance measurements are mainly related to the capacitance value register, the excitation mode register, the measurement channel registers, the measurement mode registers, on-chip compensation setting registers.

The design of hardware circuit. AD7746 uses an I2C serial interface which is easy to be 
controlled and corrected by the single chip microcomputer. The design is composed of interdigital capacitance sensor, capacitance detection chip, a microcontroller and a LCD display.The system block diagram is shown in figure 4.

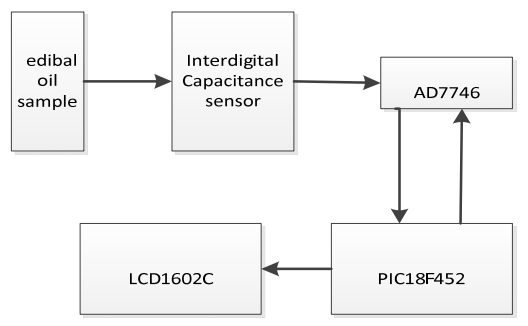

Fig.4 Flow chart of hardware detection device

MCU select PIC18F452 singlechip with the I2C serial interface,PIC18F452 is a low-power consumption, anti-interference ability, internal resource-rich, high-performance reduced instruction set bit microcontrollers, 16-bit wide instructions, 8-bit wide data. Its instruction compatible PIC16 series and PIC17 microcontrollers. Internal 32K FLASH, 1.5K RAM, the oscillation frequency up to $40 \mathrm{M} \mathrm{Hz}, 8$ channel 10-bit A / D, sampling rate, programmable [4].

PIC18F452 microcontroller sets internal registers on AD7746 and reads the capacitance value of measure, further carries on the data analysis and processing, through LCD1602C displayed.In floating measurement single-ended mode, the input "CIN" and incentive "EXC" of AD7746 capacitor are respectively measured to bipolar plate interdigital capacitor which is under test. The AD7746 measured capacitance $\mathrm{C}$ contains plate capacitor $\mathrm{C} 1$ which is under test and the additional capacitor $\mathrm{C} 2$, namely

$$
C_{1}=C-C_{2}
$$

Among them,the additional capacitance value $\mathrm{C} 2$ can be measured at the plate junction capacitance disconnected.Though the AD7746 has programmable compensation ability, in order to guarantee the system reliable operation and high precision measurement, but also to solve problems such as peripheral circuit interference and shielding [5].

Serial communication software implementation.This design uses the Microchip company for its PIC series microcontroller specially equipped MPLAB integrated development environment, using PIC series microcontroller $C$ language in the MPLAB IDE V8.60 integrated development environment realization the debugging .

Software design idea of the Edible oil detection device is to use software to replace and simplify the hardware, use the hardware circuit and software technology basically meet the requirement of system function that integration and easy to modify. After successfully built a relatively simple hardware circuit, the main function of edible oil detection device is to rely on software design completed.

AD7746 consists of 19 8-bit registers, each register has its own specific address, if you want a writeable registers for parameter modification, just write operation according to its address, numerical will be modified in writing. AD7746 register read and write should be strictly in accordance with the device to the read and write timing.Read/write sequence is shown in figure 5 .

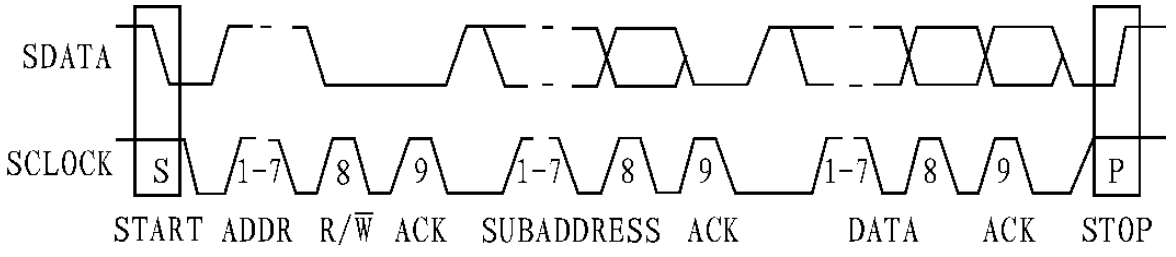


Fig.5 Sequence diagram of data transfer based on AD7746

In data transmission, SDA carries the data, SCL synchronize.Both read and write, what sent to the SDA line must be 8 each byte, each byte must be followed by an acknowledge bit.But each transmission can sent an unlimited number of bytes. The data what is transmitted is mainly composed by the start signal, the address code, a number of data bytes, and an acknowledge bit and a stop signal .In the process of data transmission, when the SCL line is high level, the SDA line must be ensure data stable. Send one byte of data, the receiver must send an acknowledge signal .Read and write device should be operated according to the figure 6 .

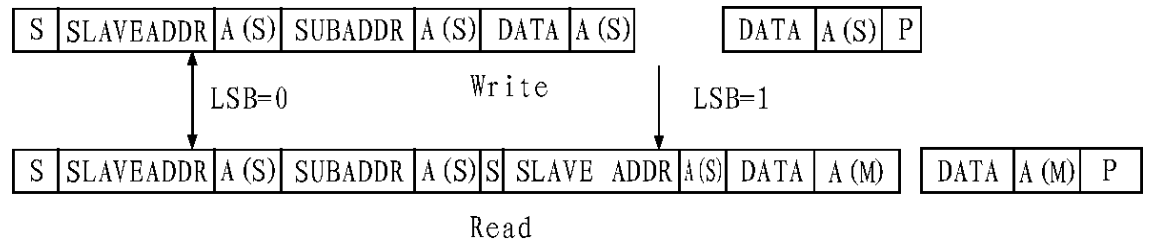

Fig.6 Logic operation figure of read and write sequence based on AD7746

During the reading, the microcontroller should first issue a start signal S,and then write to the AD7746 the write address SLAVEADDR.Microcontroller receives the response signal A (S), and then writes to the AD7746 the address SUBADDR that is need for register. When the microcontroller again receives the response A (S), and secondly launchs a start signal S,and then writes read address SLAVEADDR. when the microcontroller received response signal (S) for the third time, A handshake signal between master and slave machine is finished, after which the slave will continuously transmit data within the device out.In the process of transmitting data, there is no need to write internal register address[6].

\section{Experiment and conclusion analysis}

First measurement of different oil products. As shown in Table 1. According to one of the smallest oil dielectric constant, in order to measure the dielectric constant of illegal oil added. As shown in Table 2.Then to measure the capacitance value of different fried times oil,the experiment use fresh peanut oil $1 \mathrm{~L}$ and chicken leg $100 \mathrm{~g}$ which are bought in the supermarket,temperature taken as $25^{\circ} \mathrm{C}$, every time the same frying temperature taken as 150 ,each frying time is controlled in half an hour.Frying process without any condiment to prevent $\mathrm{Na}$ metal ion effects on measurement results.Fried chicken, respectively 1, 2, 3 times, 4 times, then remove the oil sample, filtered, cooled to room temperature, into a container prepared in advance for use.

Tab.1 Capacitance measured value of different kinds of oil

\begin{tabular}{cc}
\hline Product types & Measured capacitance values $(\mathbf{p F})$ \\
\hline Peanut oil & 2.43 \\
Corn oil & 2.52 \\
Camellia oil & 2.67 \\
Wheat germ oil & 2.92 \\
Sesame oil & 3.05 \\
Blend oil & 3.15 \\
\hline
\end{tabular}


Tab.2 Measured value of adding recycled waste oil

\begin{tabular}{ccc}
\hline Peanut oil dosage (drop) & $\begin{array}{c}\text { The first capacitance } \\
\text { measured value }(\mathrm{pF})\end{array}$ & $\begin{array}{c}\text { The second capacitance } \\
\text { measured value }(\mathrm{pF})\end{array}$ \\
\hline 1 & 2.49 & 2.50 \\
2 & 2.53 & 2.55 \\
3 & 2.69 & 2.70 \\
4 & 2.74 & 2.78 \\
\hline
\end{tabular}

Tab.3 Measured value of fried oil

\begin{tabular}{ccc}
\hline Frying times & $\begin{array}{c}\text { The first capacitance } \\
\text { measured value }(\mathrm{pF})\end{array}$ & $\begin{array}{c}\text { The second capacitance } \\
\text { measured value }(\mathrm{pF})\end{array}$ \\
\hline 1 & 3.11 & 3.12 \\
2 & 3.23 & 3.24 \\
3 & 3.30 & 3.32 \\
4 & 3.35 & 3.37 \\
\hline
\end{tabular}

In a conclusion,different oil products has different capacitor values, by detecting different types of edible oil,we found that the peanut oil has the minimum capacitance value and the cooking oil has the maximum capacitance value, which is often described that the cooking oil is mixed with other oil ingredients and has more polar molecules. Peanut oil has better quality,mixed with less ingredients and polar molecules. We also added the waste oil to peanut oil which has smaller capacitance value and measured the capacitance value.The results illustrated that the capacitance value increased with the addition of the waste oil droplets.After heat treatment after frying, the capacitance value generally increases with increase in the number frying. From a health perspective, frying time increase, then polarized molecules increase, there is a potential threat to the health when beyond a certain scope. Thus oil frying time and frequency should be reduced as far as possible, cooking oil processing and oil should be replaced periodically, don't fry for a long time.

\section{References}

[1] Han Boxian. Four plate capacitance sensor application research in the micro displacement measurement . Hebei, hebei university of technology(2005)

[2] Lu Xin,He Mingxuan,et al. Design of capacitance detecting system based on capacitive sensing probe.Transducer and Microsystem Technologies.30(12),2011,pp.95-101.

[3] Ma Jiangang,Zhang Tianhong,Zhu Lihua. Study on capacitance method clearance measurement application system based on AD7746. Transducer and Microsystem Technologies.27(2),(2008) 65-70.

[4] Light faming. PIC microcontroller programming . Beijing: science press. (2010) 123-139.

[5] Chen Xinjian. PIC single chip microcomputer application development tool making with the experiment . Beijing: Beijing aviation college.(2006) 145-158.

[6] Ma Jiangang. Application system research. Nanjing: nanjing university of aeronautics and astronautics(2006) 